\title{
Design of a high-speed electrochemical scanning tunneling microscope
}

Y. I. Yanson, F. Schenkel, and M. J. Rost

Citation: Review of Scientific Instruments 84, 023702 (2013); doi: 10.1063/1.4779086

View online: http://dx.doi.org/10.1063/1.4779086

View Table of Contents: http://aip.scitation.org/toc/rsi/84/2

Published by the American Institute of Physics

\section{Articles you may be interested in}

A high stability and repeatability electrochemical scanning tunneling microscope

Review of Scientific Instruments 85, 125103 (2014); 10.1063/1.4902975

A new and sophisticated electrochemical scanning tunneling microscope design for the investigation of potentiodynamic processes

Review of Scientific Instruments 70, 3641 (1999); 10.1063/1.1149971

A nanopositioner for scanning probe microscopy: The KoalaDrive

Review of Scientific Instruments 83, 023703 (2012); 10.1063/1.3681444

A flexible, highly stable electrochemical scanning probe microscope for nanoscale studies at the solid-liquid interface

Review of Scientific Instruments 79, 103701 (2008); 10.1063/1.2992480

Scanning probe microscopes go video rate and beyond

Review of Scientific Instruments 76, 053710 (2005); 10.1063/1.1915288

Design of a scanning tunneling microscope for electrochemical applications

Review of Scientific Instruments 59, 2333 (1998); 10.1063/1.1139957

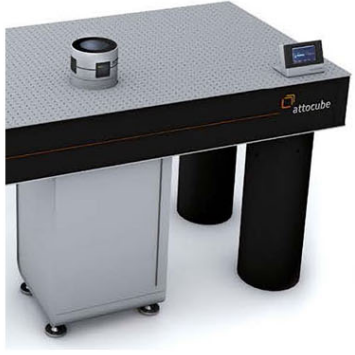

Obstruction free access

optical table with integrated cryocooler

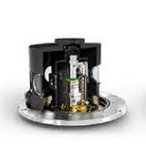

Various Objective 0ptions

\section{attoDRY800}

- Cryogenic Temperatures

- Ultra-Low Vibration

- Optical Table Included

- Fast Cooldown
$5 \%$ DISCOUNT

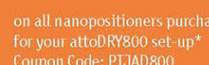




\title{
Design of a high-speed electrochemical scanning tunneling microscope
}

\author{
Y. I. Yanson, F. Schenkel, and M. J. Rost ${ }^{\mathrm{a})}$ \\ Kamerlingh Onnes Laboratory, Leiden University, Niels Bohrweg 2, 2333 CA Leiden, The Netherlands
}

(Received 26 September 2012; accepted 4 January 2013; published online 5 February 2013)

\begin{abstract}
In this paper, we present a bottom-up approach to designing and constructing a high-speed electrochemical scanning tunneling microscope (EC-STM). Using finite element analysis (FEA) calculations of the frequency response of the whole mechanical loop of the STM, we analyzed several geometries to find the most stable one that could facilitate fast scanning. To test the FEA results, we conducted measurements of the vibration amplitudes using a prototype STM setup. Based on the FEA analysis and the measurement results, we identified the potentially most disturbing vibration modes that could impair fast scanning. By modifying the design of some parts of the EC-STM, we reduced the amplitudes as well as increased the resonance frequencies of these modes. Additionally, we designed and constructed an electrochemical flow-cell that allows STM imaging in a flowing electrolyte, and built a bi-potentiostat to achieve electrochemical potential control during the measurements. Finally, we present STM images acquired during high-speed imaging in air as well as in an electrochemical environment using our newly-developed EC-STM. () 2013 American Institute of Physics. [http://dx.doi.org/10.1063/1.4779086]
\end{abstract}

\section{INTRODUCTION}

One of the limitations of conventional scanning probe microscopes (SPMs) is their rather slow imaging speed. It takes tens of seconds to several minutes to acquire an image with a conventional scanning tunneling microscope (STM). These speeds are enough for imaging static structures or very slow dynamics, such as those observed at low temperatures. However, many relevant processes happen at higher temperatures and, therefore, at much higher rates.

In recent years, much progress has been made in the development of high-speed SPMs (for an overview please see Refs. 1-4 and references therein). In particular, it was shown that high-speed imaging in electrochemical environment using electrochemical STM (EC-STM) can provide valuable information about the dynamics of processes that occur at an electrode surface. ${ }^{5-8,32}$ One reason for the rapid development of the high-speed SPMs is that fast data acquisition and processing systems, high gain high bandwidth tunneling current preamplifiers, and high speed feedback electronics have become available. ${ }^{9,10}$ However, not every SPM that is equipped with fast electronics is capable of scanning with high speeds. The reason for that are mechanical resonances of different SPM components, especially in the mechanical loop that mechanically couples the tip with the sample. These resonances usually occur at frequencies ranging from several hundreds of hertz to several kilohertz. Even if the scanning motion frequency is much lower than one of the mechanical resonance frequencies, the resonance may be excited, since scanning is commonly performed with a triangular waveform. ${ }^{9}$ This could lead to the loss of imaging quality and in the worst case result in a severe damage of the scanning probe or the sample. Thus, for designing a fast SPM, it is important to optimize the mechanical loop such that the mechanical resonances are

a) rost@physics.leidenuniv.nl. shifted to higher frequencies or that the vibration amplitudes at the resonances are below a certain threshold.

It has been demonstrated that finite element analysis (FEA) calculations can be successfully applied for the calculation and optimization of mechanical resonances of an SPM, see, e.g., Refs. 11-15. Usually, FEA has been used for the determination of vibration eigenfrequencies of different mechanical components of a microscope. However, a mechanical resonance of a part of the mechanical loop may not always have a significant effect on the imaging quality. For example, for obtaining atomic resolution one would like the mechanical loop to be stable at the range of at least $10^{-10} \mathrm{~m}$ in the longitudinal direction (along the $\mathrm{X}$ - or Y-axis) and at least $10^{-11} \mathrm{~m}$ in the lateral direction (Z-axis). If a mechanical resonance is excited and its amplitude is larger or comparable with these ranges, it will lead to the loss of imaging quality. On the other hand, if the amplitude of the resonance is lower than those values, e.g., $10^{-12} \mathrm{~m}$, no significant distortion will be introduced into the measurements. In order to obtain the relative displacement of the scanning probe and the sample surface, eigenfrequency calculations are not sufficient. Instead, a simulation of the whole mechanical loop as a function of different scanning frequencies is required. Also, damping of the components of the mechanical loop has to be taken into account in such a simulation. Despite the importance for designing an SPM, the reports on calculations of the frequency response of the complete mechanical loop of an SPM are limited. ${ }^{14-16}$

In this paper, we describe a bottom-up approach of constructing a fast EC-STM by implementing FEA calculations of the whole mechanical loop excited by the scanning motion of the piezo element. Based on primary results of these calculations, we manufactured a prototype STM. We characterized the vibrational response of the prototype setup using an optical vibrometer with sub-ångström resolution. Based on these results, we optimized the FEA model and manufactured the real, final STM. We show first results measured with our 
EC-STM, which have met our expectations from the FEA simulations.

\section{CONSTRUCTION OF THE STM}

\section{A. General design considerations}

\section{Coarse approach mechanism}

To conduct STM measurements, a conducting tip has to be brought stable to a distance of $<10^{-9} \mathrm{~m}$ from the sample. This can be achieved by a piezo tube, which is also used for the scanning motion. However, piezo tubes have only a very limited extension distance, ranging from several hundreds of nanometers to tens of micrometers. This implies that the tip first has to be brought close to the surface at a distance within the piezo range. This process is called coarse approach. Many different types of coarse approach mechanisms have been proposed and employed in different STM designs. It is important to realize that the coarse approach mechanism is usually a part of the mechanical loop of an STM. Thus, choosing the optimal coarse approach is crucial for the high-speed performance of an STM. In fact, our FEA calculations as well as calculations by other group members showed that the coarse approach is very often the most susceptible part of the mechanical loop. ${ }^{14,15}$

The coarse approach mechanisms can be divided into two groups: those containing piezo elements (either tubes, or stacks), and those consisting of an electrical motor and screws and/or flexible hinges. If one looks at material properties of different piezo materials and compares them with the material properties of, e.g., stainless steel, it is obvious that a system containing no piezo elements in the mechanical loop would be the stiffest one. Such a choice will have higher resonance frequencies. Thus, for our design, we have decided to use micrometer screws coupled to stepper motors with gear boxes as the coarse approach mechanism. In fact, using flexible hinges could be even more advantageous for the mechanical stability, but their limited range is a disadvantage.

\section{Geometry}

Based on our choice of the coarse approach mechanism, we have considered two possible basic geometries. The first geometry, which we call the "standing head," corresponds to the scanner head resting on the micrometer screws of the coarse approach, see Fig. 1(a). The screws are rigidly attached to the base of the microscope and the sample is also located on the base. As the base can be firmly attached to a bulk mass of an STM holder or housing, it can be assumed as stationary in further FEA simulations. In the "hanging sample" geometry, the base plate, on which the sample is located, is hanging on the coarse approach screws. The screws are mounted into the scanner head and the latter is mounted into a rigid holder. This geometry is presented in Fig. 1(b).

Both geometries were evaluated using FEA calculations for resonance frequencies as well as for the frequencydependent response of the tip-sample displacement, ${ }^{17}$ see Fig. 2. In general, FEA calculations of the hanging sample (a)

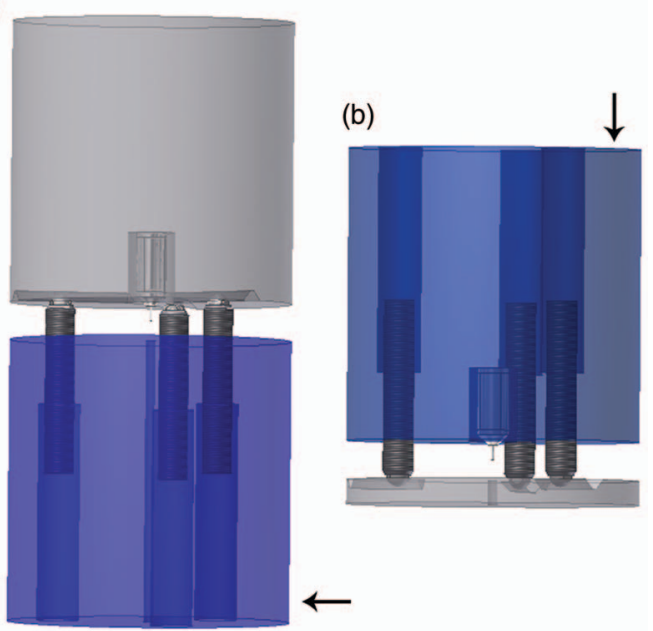

FIG. 1. Models of two geometries considered for the STM: (a) the "standing head" and (b) the "hanging sample." The part that can be rigidly clamped in a holder is imaged in blue and marked by arrows.
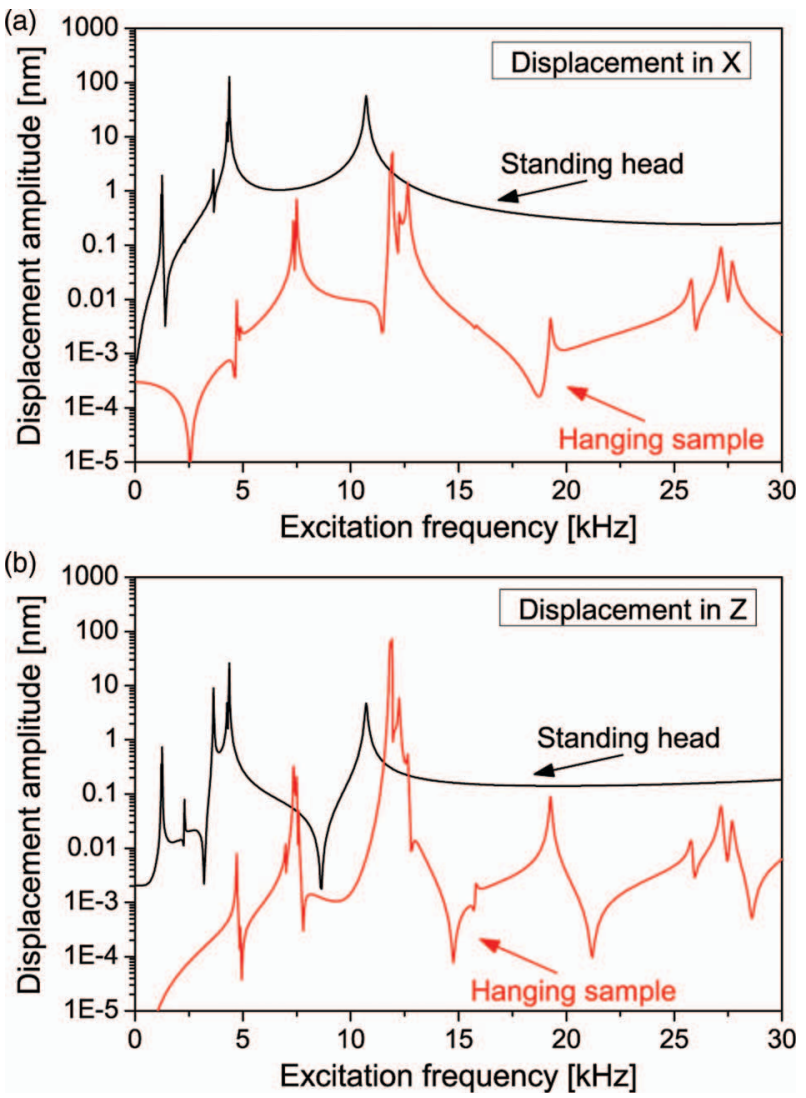

FIG. 2. Simulated frequency response of the relative tip-sample displacement, calculated using FEA, of the two geometries on the piezo tube motion at different frequencies. The "standing head" geometry is represented by the black line and the "hanging sample" geometry is represented by the red line. In these simulations, the piezo was excited with a sine waveform in the $\mathrm{x}$-direction with a peak-to-peak amplitude of $51 \mathrm{~V}$. This corresponds to a scan distance of $180 \mathrm{~nm}$ at the end of the tip holder capillary. For a usual tip length of $\sim 8 \mathrm{~mm}$, the scan distance would be $\sim 380 \mathrm{~nm}$. The relative displacement amplitudes minus the non-resonant displacement of the tip in the $\mathrm{x}$-direction (a) and the $\mathrm{z}$-direction (b) are plotted. The $\mathrm{x}$-direction is along the scan direction and the $\mathrm{z}$-direction is perpendicular to the scan plane. 


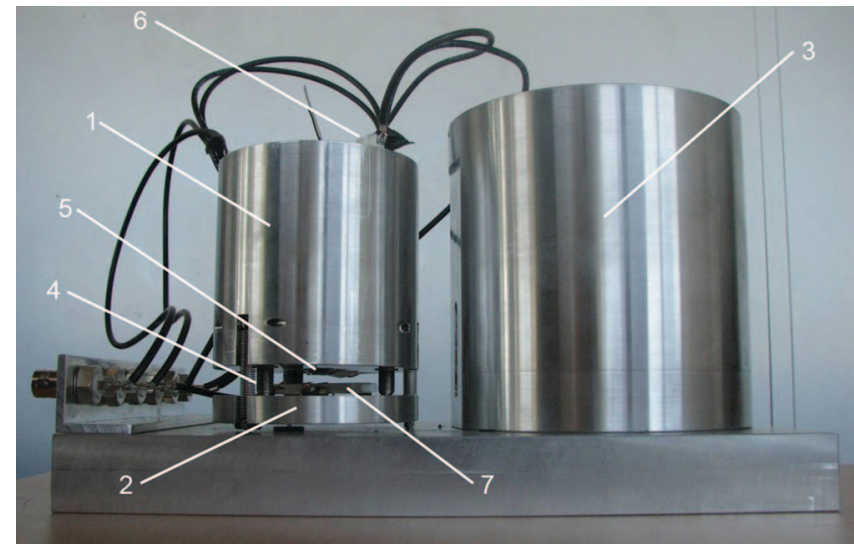

FIG. 3. Photo of the prototype STM. The components are: (1) scanner head; (2) sample plate; (3) STM holder; (4) micrometere screws and springs; (5) piezo tube with the tip holder; (6) connectors for the electrodes on the piezo tube; (7) flow cell, mounted on the sample plate.

geometry show a much better result. First, the frequency of the first resonance is much higher, as compared to the standing head geometry. Second, the amplitude at the first resonance is several orders of magnitude lower. ${ }^{18}$ In addition, in the hanging sample geometry, the first resonance corresponds not to the bending mode of the screws, but to the bending of the sample plate. Thus, the resonance frequency of this mode could be easily increased and also the response could be lowered by changing the geometry of the sample plate, making it reinforced by perpendicular blades, see Sec. II C.

Based on these FEA calculations, we conclude that the hanging sample geometry is generally more suitable for fastspeed STM, showing higher resonance frequencies and lower response at resonances as the standing head geometry.

\section{B. Prototype setup}

For testing the validity of the FEA calculations, we constructed a prototype STM setup as shown in Fig. 3. Based on our FEA calculations result, we have chosen for the "hanging sample" geometry. The scanner head of the prototype setup is made of aluminum. A piezo tube made of EBL\#2 material is glued in the center. ${ }^{19}$ The length $(\mathrm{L})$ of the tube is $12.7 \mathrm{~mm}$, the outer diameter (OD) is $6.35 \mathrm{~mm}$, and the inner diameter (ID) is $4.83 \mathrm{~mm}$. The four outer electrodes of the tube and the inner one are contacted by wires, which are attached to connectors on the top side of the scanner head. A boron nitride tip holder with two discs made of tantalum foil and a stainless steel capillary are glued to the end of the piezo tube. The capillary of the tip holder sticks out $\sim 3 \mathrm{~mm}$ above the boron nitride ceramics assembly, which has a height of $3 \mathrm{~mm}$. A wire is glued to the lower tantalum shield. A coax cable is glued such that the inner wire is connected to the steel capillary and the shield is glued to the upper tantalum shield. The piezo tube is coated with a polyurethane coating ${ }^{20}$ to protect it from moisture and to make it suitable for electrochemical measurements. The same configuration of the piezo tube and the tip holder was afterwards assembled for the final version of the fast EC-STM (see below). Three brass bushings are rigidly pressed into the scanner head. Micrometer screws with

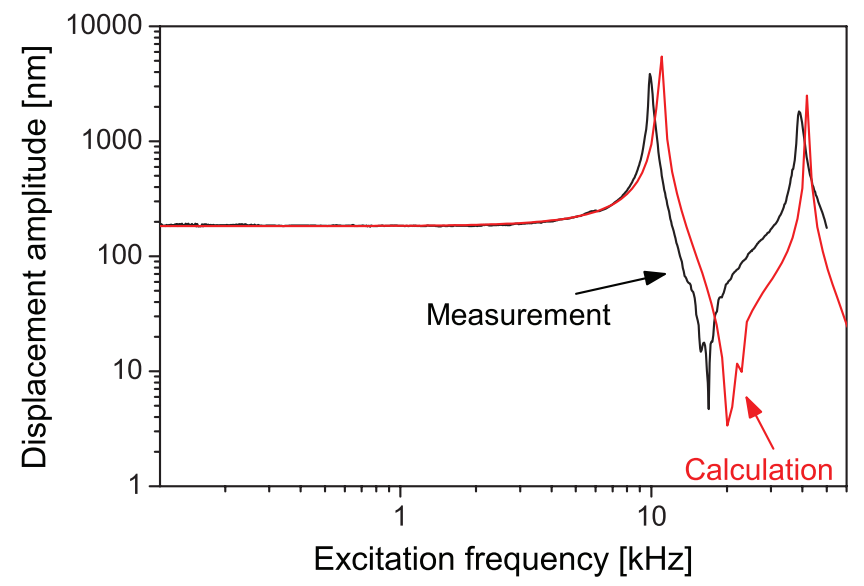

FIG. 4. Measured (black line) and calculated (red line) frequency response of the EBL\#2 piezo tube (6.35 $\mathrm{mm}$ OD, $4.83 \mathrm{~mm} \mathrm{ID,} 12.7 \mathrm{~mm} \mathrm{~L}$ ) with the tip holder. The displacement is measured and calculated at the end of a capillary that sticks $3 \mathrm{~mm}$ above the ceramic tip holder, which has a height of $3 \mathrm{~mm}$. The piezo tube was excited with a $51 \mathrm{~V}$ peak-peak amplitude sine waveform.

ball-shaped tips ${ }^{21}$ are inserted into the bushings. The sample plate is also made of aluminum. Four flat springs are attached to the plate for mounting a liquid cell onto it. Three v-shaped grooves are cut into the plate. The sample plate is attached to the scanner head by placing the micrometer screws into the groves and pulling the plate to the head by three springs. To rigidly fix the test setup, a massive holder made of aluminum is used. The scanner head is inserted into the holder and clamped by fixing screws.

The prototype setup was used to measure the mechanical response at different spots of the sample plate and the sample to the vibrations excited by the piezo motion. For the vibration measurements, an optical vibrometer was used. ${ }^{22}$ The piezo tube was excited by a sine wave with a $51 \mathrm{~V}$ peakpeak amplitude. The frequency was changed stepwise and at every excitation frequency, the displacement amplitude was measured using a lock-in type of measurement. For the measurements, the internal oscillator of the spectrum analyzer (Agilent 4395B) was used as the sine waveform source. The signal was amplified by the piezo drivers (LPM) and supplied to the electrodes of the piezo tube. The mechanical displacement of the sample or sample plate was measured using an optical vibrometer. The output of the vibrometer was fed to the input of the spectrum analyzer.

For calibration purposes and to check the validity of the FEA model of the piezo tube, we measured the mechanical response on the STM tip holder. The comparison between the measurements and the calculation is shown in Fig. 4. The calculated displacement amplitude matches well with the measured displacement both off the resonance and at the resonance frequency. The resonance peak at $9853 \mathrm{~Hz}$ in the measurement appears at slightly lower frequency as compared to the calculation. The calculated resonance is located at $11014 \mathrm{~Hz}$. This difference can be explained by the imperfections of the piezo material and also by a slightly different geometry and mass of the real STM tip holder as compared to the model.

Next, we measured the displacement amplitudes at different parts of the prototype STM as a function of the piezo 


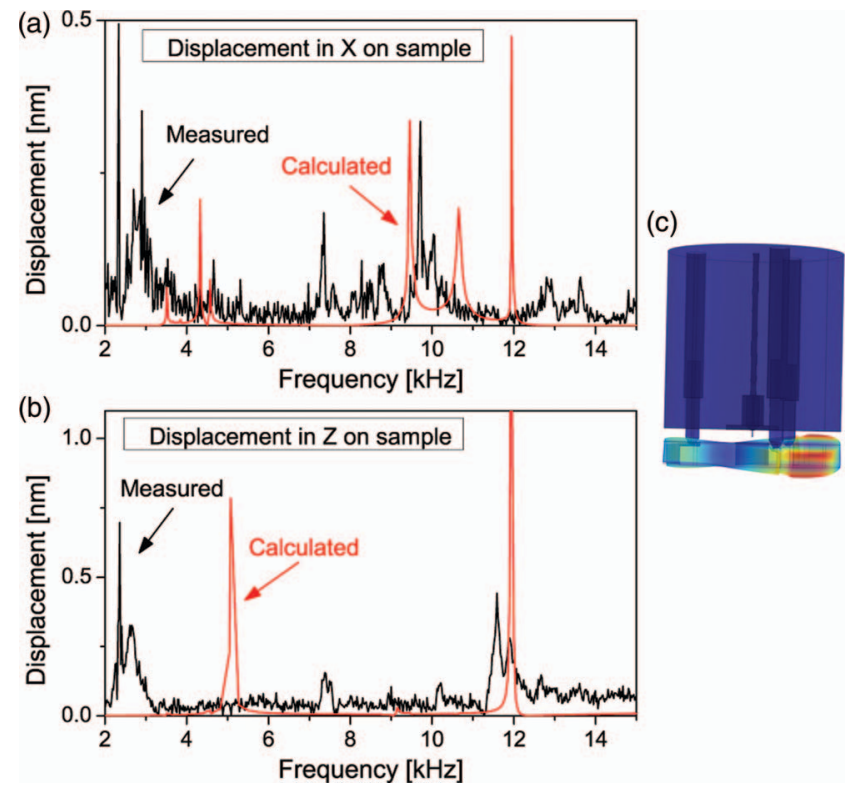

FIG. 5. Frequency response of the prototype STM on the vibrations excited by the piezo motion: comparison between the laser vibrometer measurements (black lines) and the FEA calculations (red lines). In (a), the piezo is excited in the x-direction, whereas in (b), it is excited in the z-direction. In both cases, the displacement is measured at the sample position. The piezo tube was excited with a $51 \mathrm{~V}$ peak-peak amplitude in the measurements as well as in the calculations. In (c), a schematic representation of the bending vibration mode of the sample plate, resulting in a resonance at about $7.5 \mathrm{kHz}$, is shown.

excitation frequency. Figures 5(a) and 5(b) show a comparison between the measured and the calculated displacement amplitudes at the sample along the $\mathrm{X}$-axis and the Z-axis, respectively. The piezo is excited in the same direction as the direction in which we measured the response. In Figs. 5(c) and 5(d), measurements and calculations at the edge of the sample plate are presented. Two resonances can be seen in both measurements that could significantly influence the scanning with the STM and lead to the loss of imaging quality, and that do not correspond to the piezo tube resonance around $10 \mathrm{kHz}$. These are the peaks around $2.6 \mathrm{kHz}$ and $7.5 \mathrm{kHz}$. The amplitudes of the other (resonance) peaks are very small $(\sim 0.1 \mathrm{~nm})$, so that these resonances will not significantly distort the STM imaging.

Although the calculated amplitudes at resonances are of the same order of magnitude as the measured values, the agreement between the positions of the measured and the calculated peaks is rather inferior. However, by a thorough analysis of the measurement results, presented in Fig. 5 with the corresponding calculations, we could identify the mode associated with the peak at $7.5 \mathrm{kHz}$. This peak corresponds to a bending vibration mode of the sample plate. This mode is shown in Fig. 5(c). The peak at $2.6 \mathrm{kHz}$ was impossible to identify with the simulations of the prototype scanner only. However, by modeling both the holder of the prototype setup and the scanner, we have found that several modes associated with the bending of the holder are located between $2 \mathrm{kHz}$ and $4 \mathrm{kHz}$. Thus, we associate the broad peak at $2.6 \mathrm{kHz}$ with the holder of the test STM.

The reason for the discrepancy between the measurements and the calculations can be due to several factors.
First, somewhat lower measured resonance frequencies and different amplitudes at resonances as compared to the calculated ones can be explained by the imperfections of the material of the prototype STM and by the damping in the real material. In fact, in the calculations a constant, frequencyindependent loss factor has been used to account for material damping. ${ }^{14,15,33}$ It is known that this is a rather rough approximation underestimating damping especially for higher frequencies. Therefore, the calculated results can be viewed as the worst case scenario in terms of the amplitude values at resonances. Second, there are some simplifications used in the model as compared to the real setup that could influence the results of the calculations. For example, the micrometer screws are assumed to be rigidly mounted into the scanner head. In reality, the screws are sitting in the corresponding brass bushings, which alters the mechanical contact as compared to the model. Also, the contacts between the hemispherical tips of the micrometer screws and the sample plate are modeled as fixed point contacts in the calculations. In the real setup, this contact area has certain lateral dimensions and is free to rotate or move along the slits in the sample holder. The fixed point contacts lead to a better mechanical contact that could influence the mechanical transfer function from the piezo tube to the sample plate.

From the characterization of the prototype STM, we conclude that the geometry behaves satisfactory up to the resonance frequency of the piezo tube. The bending mode of the sample plate is the only vibrational mode below the piezo tube resonance that might affect the actual STM scanning at high frequencies. This mode is not a problem in practice, as its resonance frequency can be raised and its amplitude lowered by using a reinforced structure (see below). Also, we can conclude that despite the differences between the measurements and the calculations, the results of the simulations can be used to test whether a certain scanner geometry is suitable for facilitating fast scanning motion.

\section{Final design}

Figures 6(a) and 6(b) show the final design of the STM scanner with two different sample plates. The scanner head is a metallic cylinder. The lower part of the outside face is tapered such as it can be mounted in the holder, which is a metallic ring with a counter cone on the inner face. Three holes are made in the side walls for optical access to the tip and the sample in the assembled STM. One of the holes is used for connecting the tunneling current wire to the current preamplifier. An EBL\#2 piezo tube with the same dimensions as in the prototype STM is glued into the scanner. A tip holder, as described in Sec. II B, is glued onto the piezo tube. Wires that contact the electrodes of the piezo tube are fed through holes in the scanner head and attached to SMB connectors, which are rigidly placed on the top of the head. Three preloaded bushings with micrometer screws ${ }^{23}$ are inserted into the head for the coarse approach. The micrometer screws are connected to stepper motors with gear heads ${ }^{24}$ by means of home-made couplings. Using such combination results in a vertical displacement of only $\sim 8 \mathrm{~nm}$ per motor step. 
(a)

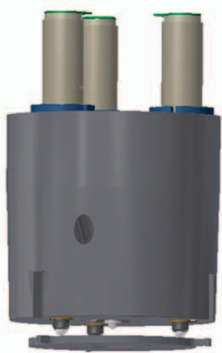

(c)

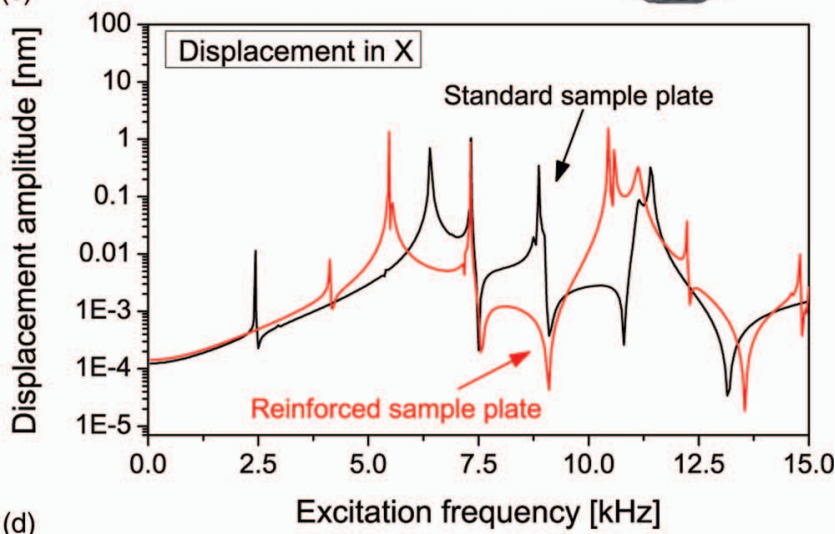

(d)

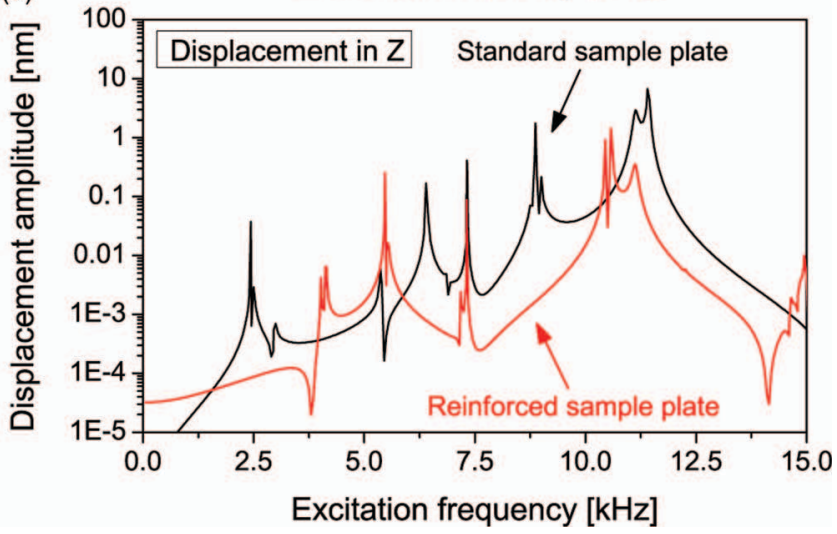

FIG. 6. Two models of the final STM scanner, equipped with (a) a standard sample plate and (b) a reinforced sample plate. Graphs in (c) and (d) show the results of FEA calculations of the displacement amplitude in $\mathrm{x}$-direction and $\mathrm{Z}$-direction of the sample as the function of the piezo excitation frequency for the simple (black) and reinforced (red) sample plates. The piezo was excited with a $51 \mathrm{~V}$ peak-peak amplitude in the same direction as the direction in which the displacement is "measured."

Two sample plates were designed and manufactured. The first sample plate is made in analogy to the plate used in the prototype STM, see Fig. 6(a). The second sample plate is reinforced at the bottom by blades that are perpendicular to the plate surface, see Fig. 6(b). The latter is fabricated to decrease the influence of the bending vibration mode of the sample plate on fast imaging by increasing the mode resonance frequency and simultaneously decreasing its amplitude. This mode was found to be the most disturbing one at frequencies lower than the resonance frequency of the piezo tube.

The results of the FEA calculations of the final scanner equipped with the two different base plates are shown in Figs. 6(c) and 6(d). As it was expected, the reinforced sample plate outperforms the non-reinforced one in its response in the $\mathrm{z}$ direction. However, the reinforced sample plate has a somewhat inferior performance in the $\mathrm{x}$ direction. The resonance peak at $5.4 \mathrm{kHz}$ found for the reinforced sample plate in the $\mathrm{x}$ direction corresponds to the bending mode of the micrometer screws. Since the mass of the reinforced plate is larger than that of the non-reinforced one, this peak appears at lower frequencies and also has a higher amplitude.

Several materials were considered for the construction of the STM. In general, the material of choice for constructing the major parts of the mechanical loop of a high-speed STM should meet the following requirements:

1. High stiffness, i.e., high Young's modulus. This increases the resonance frequencies of the mechanical loop and also makes the STM less susceptible to external vibrations.

2. High thermal stability, i.e., low thermal expansion coefficients. This is required to minimize thermal drift during measurements.

3. High damping coefficient. This leads to the lowering of the quality factor, thus lowering the vibration amplitudes at the resonances. In other words, even though resonances of some parts in mechanical loop might occur at relatively low frequencies, the vibration amplitude at resonance might be so low that it will not be an issue for imaging.

There is no material that satisfies all of the requirements listed above. However, we have considered several materials that have advantages in one or several of the properties. Our three candidates were molybdenum due to its very high stiffness, Invar due to its high stiffness and high thermal stability, and NiTi alloy (Nitinol) due to its high stiffness and high material damping. We have compared the effect of the different materials on the response of the mechanical loop using FEA calculations of the same geometry with different material properties. The calculations showed that the increased stiffness of molybdenum would not give a large advantage as compared to Invar, at least for our geometry. Further, our calculations showed that using a material with high damping such as Nitinol could be beneficial, as it would reduce the amplitudes at resonances. However, the damping properties of parts fabricated of Nitinol can depend on the alloy preparation and machining conditions. Moreover, Nitinol, which is a memory alloy, could lead to a significant stability problem of the STM at temperatures close to the martensitic transformation temperature of the alloy. This transformation temperature depends on the precise alloy composition, and is close to room temperature. We have decided to build our STM from Invar, as its high thermal stability provides a large advantage for STM imaging.

\section{Flow cell}

We designed a special liquid cell to allow for electrolyte flow and exchange in situ during actual STM measurements, see Fig. 7. We chose an elongated shape of the liquid cell, as opposed to the commonly used circular cells, such that the liquid flow at the position of the STM tip and the sample is laminar. This is necessary to minimize the influence of flow (turbulences) on the imaging quality. To improve the liquid flow characteristics even further, the liquid cell has to be made 

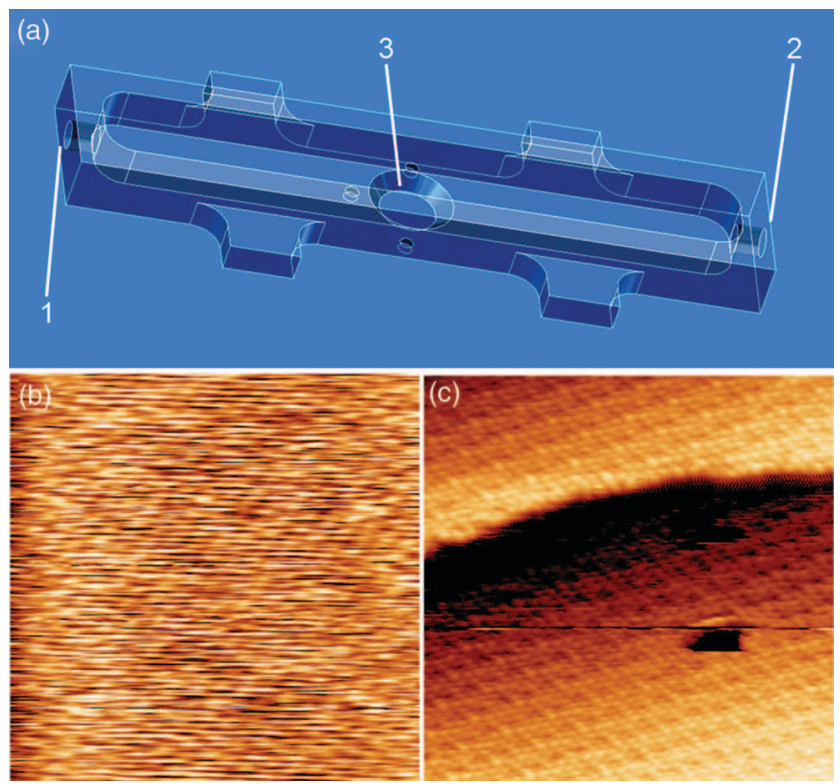

FIG. 7. Design of the flow-cell (a) and STM images measured during flow (b)-(c). (1) and (2) are the inlet and the outlet for the electrolyte. (3) is the hole for the sample. (b) Atomically resolved HOPG surface in ultra-pure water with the pump switched on at $70 \mu \mathrm{L} / \mathrm{s}$ pumping rate. The image size is $5 \times 5 \mathrm{~nm}^{2}$. (c) Cu UPD layer on the Au(111) surface imaged with the pump switched on at $100 \mu \mathrm{L} / \mathrm{s}$ pumping rate. The image size is $15 \times 15 \mathrm{~nm}^{2}$. The total height scale (from black to white) is: (b) $0.84 \mathrm{~nm}$, (c) $0.36 \mathrm{~nm}$. The tunneling parameters are: (b) $1.5 \mathrm{nA}$ tunneling current and $100 \mathrm{mV}$ tip bias; (c) $1.5 \mathrm{nA}$ tunneling current, $50 \mathrm{mV}$ tip potential, and $250 \mathrm{mV}$ sample potential.

of a hydrophilic material. We fabricated two liquid cells out of the following two materials: quartz, which has a nearly perfect chemical resistance, and Zerodur, ${ }^{25}$ which has a low thermal expansion coefficient and also good chemical inertness.

To create a liquid flow in the system, we decided to use a low flow rate peristaltic pump with multiple channels. ${ }^{26}$ One channel supplies the flow of an electrolyte into the cell from a liquid container. The second channel removes the excess electrolyte from the liquid cell, thus preventing spill over. Due to identical flow rates at different channels of the pump, a constant level of the electrolyte is maintained in the flow cell. The pump is connected to the cell via Teflon tubing. ${ }^{27}$

In general, peristaltic pumps are known to cause pulsations in the liquid systems. To test whether our pump would create pulsations that would influence the STM imaging, we conducted trial measurements with a commercial STM from molecular imaging (MI). ${ }^{28}$ For that, an HOPG single crystal was placed onto the original MI sample plate and then our flow cell was placed on top. The cell was filled with ultrapure water. A cut PtIr tip was used for STM imaging. As one can see in Fig. 7(b), the atomic resolution is obtained while pumping at a rate of $70 \mu \mathrm{L} / \mathrm{min}$. Another example of a surface imaged in flowing electrolyte at a rate of $100 \mu \mathrm{L} / \mathrm{min}$ is presented in Fig. 7(c), where the $\mathrm{Cu}$ underpotential deposition layer is imaged on the $\mathrm{Au}(111)$ surface in an electrolyte containing $0.1 \mathrm{M} \mathrm{H}_{2} \mathrm{SO}_{4}, 10 \mathrm{mM} \mathrm{CuSO}_{4}$, and $1 \mathrm{mM} \mathrm{HCl}$. We conclude that the influence of pumping on the imaging quality is negligibly small at low pumping speeds using our flow cell. At higher pumping speeds, the pulsations due to the pumping may become visible and even lead to loss of imag- ing resolution. In our experiments, we saw that the pumping rate, at which the pulsations start to influence the STM measurements, depends on different parameters such as the tubing type and length, electrolyte level in the cell, STM tip length, thickness of the tip coating, etc. Thus, we cannot define a universal maximum pumping rate, at which the STM imaging would be possible. In our experiments, we usually set the flow rate to approximately $100 \mu \mathrm{L} / \mathrm{min}$, which corresponds to a complete exchange of the electrolyte in the flow cell every 4 $\min$. At this flow rate, we see no measurable influence of the flow on the imaging quality.

Finally, we would like to mention that although it is possible to maintain constant flow in the cell, transport of the electrolyte (or ions in the electrolyte) towards the sample proceeds via diffusion, since the sample is located in a depression relative to the plane of the cell in which laminar flow is maintained. Thus, in all deposition experiments conducted in the flow-cell, one of which is described in Sec. III C, the mass transport of ions towards the sample happens via diffusion and convection can be neglected. We estimate the time that is needed for the concentration at the sample surface to reach the value in the bulk of electrolyte to be $\sim 1 \mathrm{~min}$ (for typical values of the diffusion coefficient of ions in water of $10^{-5} \mathrm{~cm}^{2} \mathrm{~s}^{-1}$ ). If a new ionic species is introduced into the electrolyte at the inlet of the flow cell, it would take $\sim 3 \mathrm{~min}$ for the concentration at the sample surface to reach the same value as at the inlet.

\section{E. STM assembly and housing}

Figure 8 shows two photos of the complete STM assembly. The STM housing consists of a scanner holder and an environmental chamber for the possibility of maintaining an inert gas atmosphere. The scanner holder is composed of an aluminium disc resting on three aluminium legs. The bars are connected to the bottom plate of the holder. An Invar ring is placed in the middle of the aluminium disc. The inner face of the ring is tapered such that if the scanner head is placed into it, it gets rigidly connected to the holder. This fixing mechanism is similar to the so-called Morse taper, found in machinery.

The environmental chamber is connected via a Viton ring to the STM holder to inhibit mechanical coupling between them and to seal the chamber for maintaining an inert gas atmosphere in it. The side frames of the chamber are connected to the bottom plate of the holder. The side walls of the environmental chamber are made of plexiglass. They are fixed to the frame via a click-in mechanism for easy removal. On the side walls of the chamber, several ports are located for electrical connections, tubing, and gas connections.

\section{PERFORMANCE}

\section{A. HOPG in air}

The surface of HOPG was imaged in air to test the performance of the video-rate EC-STM. A freshly cleaved HOPG crystal was mounted onto the reinforced base plate and a cut PtIr tip was used for imaging. Figure 9(a) shows an atomically 

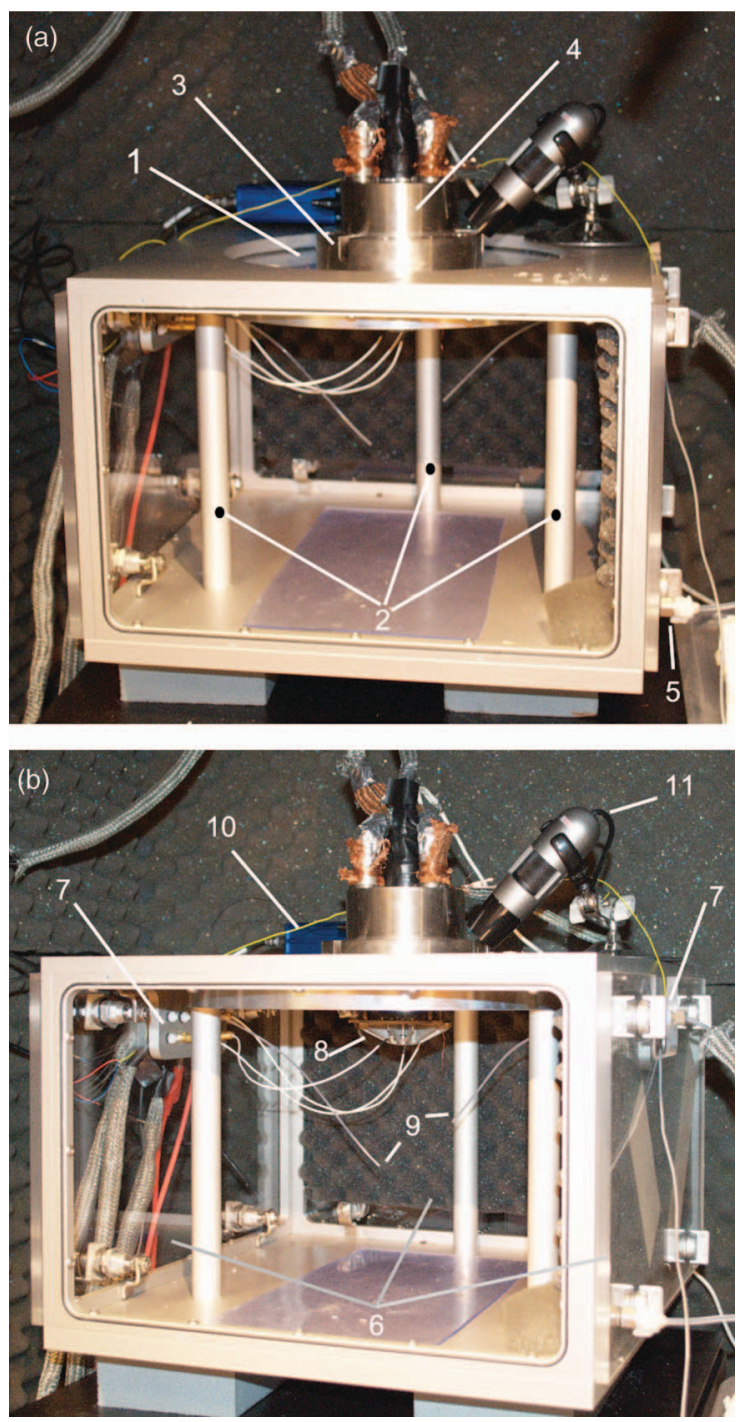

FIG. 8. Photos of the final STM assembly. The STM scanner holder consists of an aluminium plate (1) that rests on three aluminium legs (2). An Invar ring in the middle of the plate (3) is used for clamping the scanner (4). The holder is connected to the environmental chamber via a Viton ring (not visible). An inert gas is supplied to the chamber via the input (5). The walls of the environmental chamber (6) are made of plexiglas. Please note that the front wall was removed in the photos. The ports in the side walls (7) are used for electrical and tubing connections. The sample plate (8) is mounted on the scanner. Teflon tubes for the electrolyte flow (9) are not connected to the flow cell in these photos. On top of the STM holder, a tunneling current preamplifier (10) and a USB microscope (11) for optical access are located.

resolved height image of the HOPG surface acquired at $1850 \mathrm{~Hz}$ line rate. It can be seen from the image that there is no distortion due to unwanted mechanical vibrations. Images in Figs. 9(b) and 9(c) were acquired at a line frequency of $7250 \mathrm{~Hz}$ in hybrid mode. ${ }^{9}$ The atomic resolution at this scan speed is still preserved, although the atoms appear slightly distorted due to the excited vibrations. The distortion on the sides of the images is due to the hysteresis of the piezo material.

\section{B. Electrodeposited Cu crystals on $\mathrm{Au}(111)$}

Our purpose of designing a fast STM is not only to scan fast on relatively flat surfaces, but also to be able to image

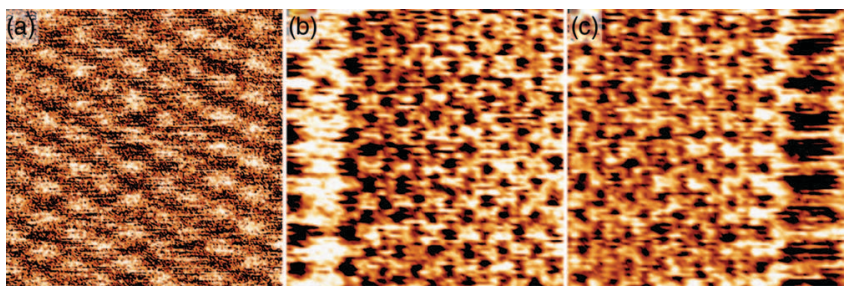

FIG. 9. STM images of HOPG in air with atomic resolution. (a) Height image acquired at $1850 \mathrm{~Hz}$ line frequency and $7.2 \mathrm{~Hz}$ image frequency; (b) and (c) are error signal images measured at $7250 \mathrm{~Hz}$ line frequency and $72.5 \mathrm{~Hz}$ image frequency. Image (b) was measured from left to right and image (c) from right to left. The size of all images is $2.4 \times 2.4 \mathrm{~nm}^{2}$. The height scale in (a) (from black to white) is $0.02 \mathrm{~nm}$. The tunneling parameters are: $\sim 3 \mathrm{nA}$ tunneling current, and $50 \mathrm{mV}$ bias voltage.

very rough surfaces. One of the situations, where a rough surface can develop, is the electrodeposition of a metal on a substrate of a different metal. In this case, growth of the metal deposit can proceed following the Volmer-Weber or StranskiKrastanov mechanisms. This can result in islands of the deposit that reach several tens to several hundreds of nanometers in height as well as in width. If one would like to image such a surface, not only fast feedback electronics and a high bandwidth preamplifier are needed, but also a stable mechanical loop of the STM with high resonance frequencies. Our EC-STM fulfills this requirement, which enables stable and relatively fast imaging of even such rough surfaces. An example of such a surface is a $\mathrm{Cu}$ bulk deposit, grown on a $\mathrm{Au}(111)$ substrate from a $0.1 \mathrm{M} \mathrm{H}_{2} \mathrm{SO}_{4}, 10 \mathrm{mM} \mathrm{CuSO}_{4}$, and $1 \mathrm{mM} \mathrm{HCl}$ solution at $-100 \mathrm{mV}$ sample potential, see Fig. 10. As one can see, we can stably image this surface, observing the $\sim 40 \mathrm{~nm}$ high $\mathrm{Cu}$ crystallites as well as resolving the monatomic steps on the $\mathrm{Au}(111)$ surface, see also Fig. 10(c).

\section{High-speed imaging during $\mathrm{Cu}$ deposition on $\mathrm{Cu}(111)$}

The ultimate purpose of our high-speed STM is to image the electrode surface with a high spatial and temporal resolution during electrochemical processes. In Ref. 32 we investigate copper electrodeposition in the presence of different additives in real-time. Another example of such a process is the $\mathrm{Cu}$ bulk electrodeposition on a $\mathrm{Cu}(111)$ surface from a solution containing $0.1 \mathrm{M} \mathrm{H}_{2} \mathrm{SO}_{4}$ and $10 \mathrm{mM} \mathrm{CuSO}_{4}$. Figure 11 shows a sequence of STM images acquired at high imaging speed during the electrodeposition of $\mathrm{Cu}$ on a $\mathrm{Cu}(111)$ crystallite, such as the ones observed in Fig. 10. From the images, one can clearly distinguish single $\mathrm{Cu}$ steps of monatomic height. At closer inspection of the images, one can see a weak corrugation on the terraces. This corrugation corresponds to the moiré pattern, which appears due to the formation of the sulfate adlayer on the $\mathrm{Cu}$ surface. ${ }^{29,30}$

Based on the STM images, we can make the following conclusions. First, the monatomic $\mathrm{Cu}$ steps tend to align themselves parallel to the high symmetry axes of the moiré pattern. Second, all steps in the images propagate with the same speed. The propagation speed is independent on the width of either the upper or the lower terraces, adjacent to the steps. This 

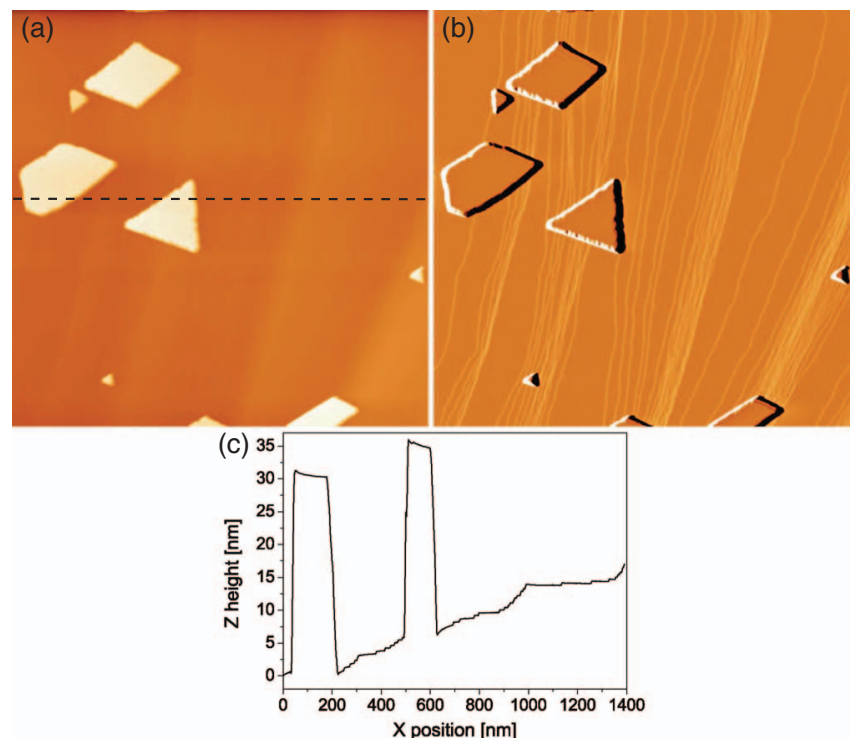

FIG. 10. STM images of $\mathrm{Cu}$ crystallites, electrodeposited on a $\mathrm{Au}(111)$ surface from a $0.1 \mathrm{M} \mathrm{H}_{2} \mathrm{SO}_{4}, 10 \mathrm{mM} \mathrm{CuSO}_{4}$, and $1 \mathrm{mM} \mathrm{HCl}$ solution at $-100 \mathrm{mV}$ sample potential vs $\mathrm{Cu} / \mathrm{Cu}^{2+}$. (a) Non-processed height image. The average height of the Cu crystallites is $\sim 40 \mathrm{~nm}$. (b) Differentiated image. $\mathrm{Cu}$ crystallites as well as atomic steps of the $\mathrm{Au}(111)$ substrate are clearly visible. The image was acquired at $2.93 \mathrm{~Hz}$ line frequency $(175 \mathrm{~s}$ per image). The size of the images is $1400 \times 1400 \mathrm{~nm}^{2}$. The tunneling parameters are: $\sim 500 \mathrm{pA}$ tunneling current, $-15 \mathrm{mV}$ tip potential, and $-5 \mathrm{mV}$ sample potential. (c) Height profile that was measured along the black line of the STM image in (a).

indicates that if the propagation of steps proceeds via the surface diffusion mechanism, then the Ehrlich Schwoebel barrier at the steps is either absent here or it plays no role in the growth mechanism at play (for a review on different modes of growth see Ref. 31 and references therein). Thus, we show that the STM imaging during electrochemical deposition can provide invaluable information about the deposition mechanism (see also Ref. 32).

\section{SUMMARY}

We have designed and constructed an EC-STM that is capable of high-speed imaging in constant current mode. In addition to its ability to image at high scan speeds, our STM is also capable of imaging very rough surfaces, such as $\mathrm{Cu}$ crystallites electrodeposited on $\mathrm{Au}(111)$, while still maintaining atomic height resolution.

The ability to image at high speed and the high stability of our EC-STM are partly due to the bottom-up approach that we used for its design. We employed FEA calculations of the frequency response of the whole mechanical loop of the STM scanner to the scanning motion of the piezo tube. Based on the results of these calculations as well as on test measurements with our prototype scanner, we were able to choose the most promising geometry of the scanner. Additionally, we optimized the geometry to increase the resonance frequency and to decrease the amplitude of the first resonance mode of the system.

In addition, we developed an electrochemical flow-cell that allows us to acquire STM images while flowing an elec-
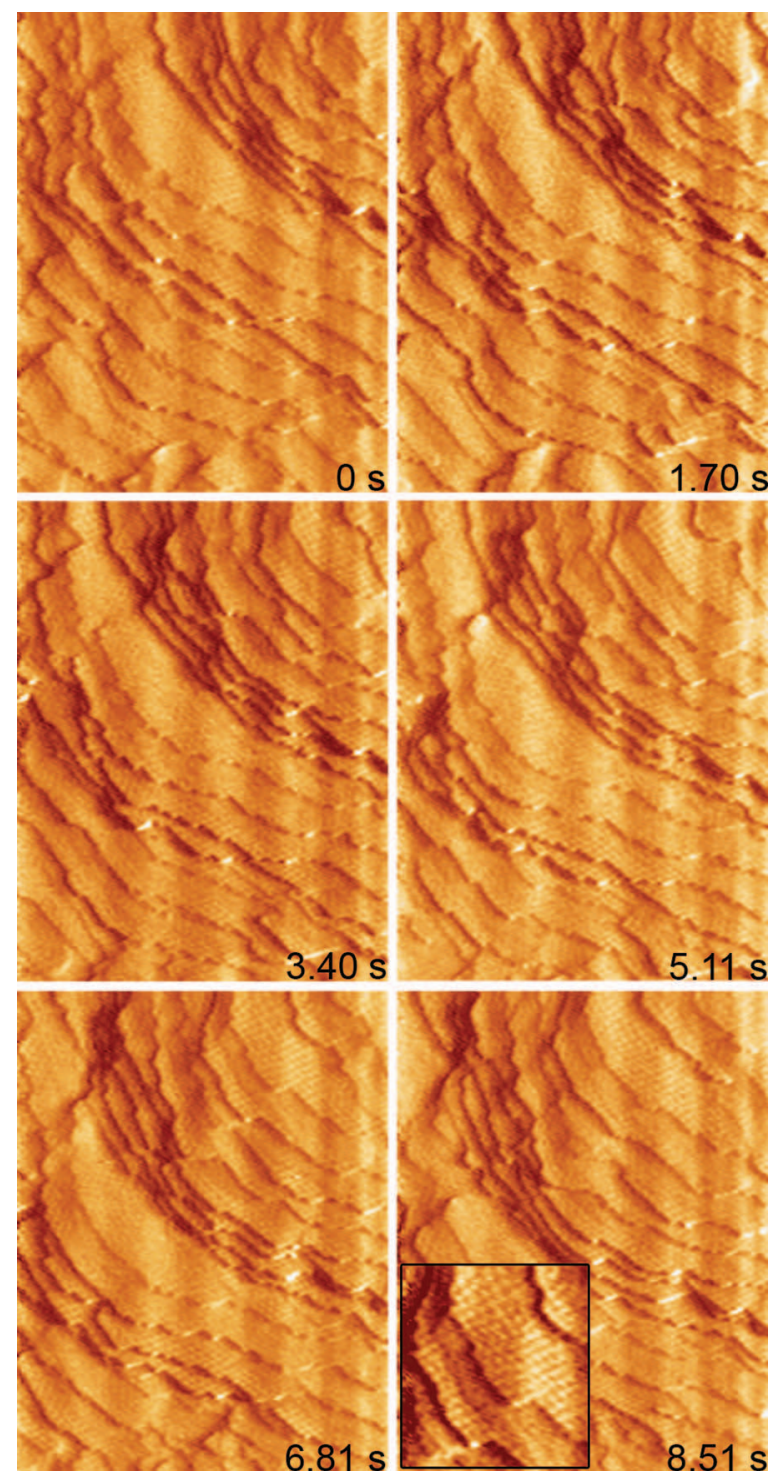

FIG. 11. Differentiated STM images of a growing $\mathrm{Cu}$ step bunch during electrodeposition on a $\mathrm{Cu}(111)$ crystallite from a $10 \mathrm{mM} \mathrm{CuSO}_{4}$ and $0.1 \mathrm{M} \mathrm{H}_{2} \mathrm{SO}_{4}$ solution at $-40 \mathrm{mV}$ sample potential vs $\mathrm{Cu} / \mathrm{Cu}^{2+}$. The images were acquired at $300 \mathrm{~Hz}$ line-rate $(0.85 \mathrm{~s}$ per image). Every second image of the acquired image sequence is shown here. The images were acquired while flowing the electrolyte. One can clearly distinguish atomic steps of the growing deposit. Also, a weak corrugation is visible on the terraces (see also inset in the last image), which corresponds to the moiré pattern that originates from the formation of a sulfate adlayer. Size of all images is $155 \times 200 \mathrm{~nm}^{2}$ and the size of the inset is $60 \times 60 \mathrm{~nm}^{2}$. The tunneling parameters are: $-5 \mathrm{mV}$ tip potential and $\sim 500 \mathrm{pA}$ tunneling current.

trolyte. This enables to, e.g., vary concentrations of species in the electrolyte or even introduce additional substances (additives) while imaging exactly the same part of the electrode surface with high resolution.

Finally, the combination of the rigid STM scanner design, the high-speed feedback, and the data acquisition electronics allowed us to reach video-rate imaging rates in constant current and hybrid modes on atomically flat surfaces of HOPG and also conduct high-speed imaging in an electrochemical environment under potential control during electrochemical deposition. This makes our EC-STM suitable for the studies of dynamic processes at an electrode surface. 


\section{ACKNOWLEDGMENTS}

We kindly would like to thank G. Schitter for giving us the possibility to conduct the optical vibrometer measurements and S. Kuiper for assistance during these measurements. The described research was supported by the Dutch Technology Foundation STW (Project No. 07720), which is the applied science division of NWO, and the Technology Program of the Ministry of Economic Affairs.

${ }^{1}$ G. Schitter and M. J. Rost, Mater. Today 11, 40-48 (2008).

${ }^{2}$ M. J. Rost, G. J. C. van Baarle, A. J. Katan, W. M. van Spengen, R. Schakel, W. A. van Loo, T. H. Oosterkamp, and J. W. M. Frenken, Asian J. Control 11, 110-129 (2009).

${ }^{3}$ F. C. Tabak, E. C. M. Disseldorp, G. H. Wortel, A. J. Katan, M. B. S. Hesselberth, T. H. Oosterkamp, J. W. M. Frenken, and W. M. van Spengen, Ultramicroscopy 110, 599-604 (2010).

${ }^{4}$ E. C. M. Disseldorp, F. C. Tabak, A. J. Katan, M. B. S. Hesselberth, T. H. Oosterkamp, J. W. M. Frenken, and W. M. van Spengen, Rev. Sci. Instrum. 81, $043702(2010)$

${ }^{5}$ O. M. Magnussen, L. Zitzler, B. Gleich, M. R. Vogt, and R. J. Behm, Electrochim. Acta 46, 3725-3733 (2001).

${ }^{6}$ O. M. Magnussen, W. Polewska, L. Zitzler, and R. J. Behm, Faraday Discuss. 121, 43-52 (2002).

${ }^{7}$ T. Tansel and O. M. Magnussen, Phys. Rev. Lett. 96, 026101 (2006).

${ }^{8}$ Y.-C. Yang, A. Taranovskyy, and O. M. Magnussen, Angew. Chem., Int. Ed. 51, 1966-1969 (2012)

${ }^{9}$ M. J. Rost, L. Crama, P. Schakel, E. van Tol, G. B. E. M. van VelzenWilliams, C. F. Overgauw, H. ter Horst, H. Dekker, B. Okhuijsen, M. Seynen, A. Vijftigschild, P. Han, A. J. Katan, K. Schoots, R. Schumm, W. van Loo, T. H. Oosterkamp, and J. W. M. Frenken, Rev. Sci. Instrum. 76, 053710 (2005).

${ }^{10}$ See http://www.leidenprobemicroscopy.com for LPM Video Rate STM Control Electronics, Leiden Probe Microscopy B.V.

${ }^{11}$ C. Braunsmann and T. E. Schaeffer, Nanotechnology 21, 225705 (2010).

${ }^{12}$ J. H. Kindt, G. E. Fantner, J. A. Cutroni, and P. K. Hansma, Ultramicroscopy 100, 259-265 (2004)
${ }^{13}$ S. C. White, U. R. Singh, and P. Wahl, Rev. Sci. Instrum. 82, 113708 (2011).

${ }^{14}$ V. Fokkema, Ph.D. dissertation, Leiden University, 2011.

${ }^{15}$ M. den Heijer, M.S. thesis, Leiden University, 2008.

${ }^{16}$ C. R. Ast, M. Assig, A. Ast, and K. Kern, Rev. Sci. Instrum. 79, 093704 (2008).

${ }^{17}$ Please note that the tip-sample displacement is defined as the displacement of the tip relative to sample minus the calculated non-resonant displacement of the tip. Using this definition, the relative displacement is zero, if no resonances occur, as we can neglect the nonresonant deformation of the mechanical loop in response to the scanning motion.

${ }^{18}$ We used the same values for damping in all calculations. We modeled damping by using a frequency-independent loss factor, which is equal to one over the quality factor, of $10^{-5}$ for the metallic parts and $10^{-2}$ for the piezo tube. ${ }^{14,15,33}$

${ }^{19}$ See http://www.eblproducts.com for EBL Products, Inc.

${ }^{20}$ See http://www.humiseal.com for HumiSeal 2A64 coating.

${ }^{21}$ See http://www.thorlabs.com for Thorlabs, Inc.

${ }^{22}$ See http://www.polytec.com for Polytec.

${ }^{23}$ See http://www.thorlabs.com for FS25AB150 screws and bushings, Thorlabs, Inc.

${ }^{24}$ See http://www.faulhaber.com for AM-1524-A-0,25-12,5-07 motors with the 15/8 zero backlash 1670:1 ration gearheads, Faulhaber Group.

${ }^{25} \mathrm{See}$ http://www.schott.com for SCHOTT AG.

${ }^{26} \mathrm{See}$ http://www.ismatec.com for IPC-N peristaltic pump, Ismatec SA.

${ }^{27}$ See http://www.idex-hs.com for IDEX Corporation.

${ }^{28}$ See http://www.home.agilent.com for MI PicoSTM, Agilent Technologies (formerly Molecular Imaging).

${ }^{29}$ M. Wilms, P. Broekmann, C. Stuhlmann, and K. Wandelt, Surf. Sci. 416, 121-140 (1998).

${ }^{30}$ P. Broekmann, M. Wilms, A. Spaenig, and K. Wandelt, Prog. Surf. Sci. 67, 59-77 (2001).

${ }^{31}$ C. Misbah, O. Pierre-Louis, and Y. Saito, Rev. Mod. Phys. 82, 981-1040 (2010).

${ }^{32}$ Y. I. Yanson and M. J. Rost, "Structural accelerating effect of chloride on cu electrodeposition," Angew. Chem., Int. Ed. (in press).

${ }^{33}$ B. Lazan, Damping of Materials and Members in Structural Mechanics (Pergamon, 1968). 